\title{
Notch1 signaling is involved in regulating Foxp3 expression in T-ALL
}

Xiaodan Luo ${ }^{1}$, Huo Tan ${ }^{1 *}$, Yueqiao Zhou' ${ }^{1}$, Tiantian Xiao ${ }^{1}$, Chunyan Wang ${ }^{1}$ and Yangqiu Li ${ }^{2}$

\begin{abstract}
Background: T-cell acute lymphoblastic leukemia (T-ALL) is a highly aggressive hematologic malignancy. Immune tolerance induced by $\mathrm{CD}^{+} \mathrm{CD} 25^{+}$regulatory T cells (Tregs) with high expression of Foxp3 is an important hypothesis for poor therapy response. Notch1 signaling is thought to be involved in the pathogenesis of this disease. Crosstalk between Notch and Foxp3 ${ }^{+}$Tregs induced immune tolerance is unknown in T-ALL. We studied Foxp3 and Notch1 expression in vivo and in vitro, and analyzed the biological characteristics of T-ALL cell line systematically after Notch inhibition and explored the crosstalk between Notch signaling and Foxp3 expression.

Methods: In vivo, we established T-ALL murine model by Jurkat cells transplantation to severe combined immunodeficiency (SCID) mice. Notch1 and Foxp3 expression was detected. In vitro, we used $\gamma$-secretase inhibitor N-S-phenyl-glycine-t-butyl ester (DAPT) to block Notch1 signaling in Jurkat cells. Notch1, Hes-1 and Foxp3 genes and protein expression were detected by PCR and western blotting, respectively. The proliferation pattern, cell cycle and viability of Jurkat cells after DAPT treatment were studied. Protein expression of Notch1 target genes including NF-KB, $p$-ERK1/2 and STAT1 were determined.

Results: We show that engraftment of Jurkat cells in SCID mice occurred in 8 of 10 samples (80\%), producing disseminated human neoplastic lymphocytes in PB, bone marrow or infiltrated organs. Notch1 and Foxp3 expression were higher in T-ALL mice than normal mice. In vitro, Jurkat cells expressed Notch1 and more Foxp3 than normal peripheral blood mononuclear cells (PBMCs) in both mRNA and protein levels. Blocking Notch1 signal by DAPT inhibited the proliferation of Jurkat cells and induced G0/G1 phase cell cycle arrest and apoptosis. Foxp3 as well as p-ERK1/2, STAT1 and NF-kB expression was down regulated after DAPT treatment.
\end{abstract}

Conclusions: These findings indicate that regulation of Foxp3 expression does involve Notch signaling, and they may cooperatively regulate T cell proliferation in T-ALL.

Keywords: Notch1, T cell, Foxp3, Leukemia

\section{Background}

T-cell acute lymphoblastic leukemia (T-ALL) is a highly aggressive hematologic malignancy that represents $10 \%$ to $15 \%$ of pediatric and $25 \%$ of adult acute lymphoblastic leukemia cases [1-6]. Compared to B-cell acute lymphoblastic leukemia (B-ALL), patients with T-ALL commonly present large tumor burdens at diagnosis and invariably poor outcomes even after intensified chemotherapy. The specific biological and molecular mechanisms that account for the aggressiveness and poor therapy response in T-ALL remain unclear and T-ALL cells induced immune

\footnotetext{
*Correspondence: tanhuo.2008@163.com

'Department of Oncology \& Hematology, the First Affiliated Hospital of

Guangzhou Medical College, Guangzhou 510230, China

Full list of author information is available at the end of the article
}

tolerance is an important hypothesis [1-6]. Some reports showed that T-ALL cells are derived from regulatory $\mathrm{T}$ cells (Tregs), which suppress the reaction of lymphocytes to tumor antigens and induce immune tolerance and malignant neoplasm progression. Foxp3 is a specific and important marker for Tregs. Recent reports showed that the aggressiveness and poor outcome of T-ALL are closely related to the large number of Tregs and high expressions of Foxp3 in tumor microenvironment $[4,7,8]$.

Notch1 is more and more concerned in T-ALL and activating mutations in the Notch1 gene are present in over $50 \%$ of human T-ALL cases making Notch1 the most prominent oncogene specifically involved in the pathogenesis of this disease [2,3,9-12]. The Notch pathway regulates

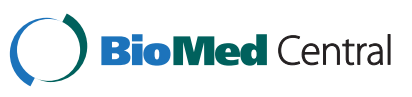

(c) 2013 Luo et al.; licensee BioMed Central Ltd. This is an Open Access article distributed under the terms of the Creative Commons Attribution License (http://creativecommons.org/licenses/by/2.0), which permits unrestricted use, distribution, and reproduction in any medium, provided the original work is properly cited. 
$\mathrm{T}$ cell proliferation and development and therefore is critical for ensuring the proper differentiation of $\mathrm{T}$ cell [3,10,12-15]. "Gain of function" mutation within Notch1 was found in both T-ALL patient samples and murine T-ALL models $[3,10,12,16]$. Activation of Notch receptors is triggered by interaction with Notch ligands Jagged and Delta-like on adjacent cells, which results in proteolytic cleavage of Notch and subsequent release of the intracellular domain (IC) $[2,3,9-12]$. Notch-IC is then transported into the nucleus and associates with $R B P-J k / C B F-1$, resulting in the activation of target genes including the Hes family of proteins [17-19]. Inhibition of Notch1 signaling using $\gamma$-secretase inhibitors (GSIs) induced rapid clearance of Notch-IC and transcriptional down regulation of Notch1 target genes. The precise mechanism by which Notch activation leads to T-ALL is still unclear. Key pathways include the PI3-kinase/Akt, mTOR and NF-kB. Zou J et al. report that Notch1 is required for hypoxia-induced proliferation, invasion and chemoresistance of T-cell acute lymphoblastic leukemia cells [1,3,14,15,20-23]. Crosstalk between Notch and these pathways is also incompletely understood and probably occurs at several levels.

Several studies have implicated the participation of Notch signaling in Treg differentiation and suppressor function. Overexpression of Notch ligand can induce Treg and Foxp $3^{+}$Tregs express high levels of Notch1 [8,24-27]. Ou-yang showed that Notch1 signaling can activate the Foxp3 promoter and Hes 1 might be an important regulatory factor at the transcriptional level in the lineage determination of Tregs development [8]. However, very few reports have shown the association between Notch1 and Foxp3 and the crosstalk between them is unknown. In this study, we show not only Notch1 and Foxp3 expression in T-ALL group both in vivo and in vitro, but also the biological characteristics of T-ALL cell line as Notch1 and Foxp3 expression was inhibited. Blocking Notch1 signaling by GSI N-S-phenylglycine-t-butyl ester (DAPT) inhibited the expressions of Notch1 and Foxp3 in Jurkat cell line, inducing apoptosis of Jurkat cells. Protein levels of NF-kB, p-ERK1/2 and STAT1 were also decreased in Notch1 inhibited Jurkat cells. These findings suggested that inhibition of Foxp3 expression does involve Notch signaling, and it may be mediated by the regulation of $N F-\kappa B, p-E R K 1 / 2$ and STAT1 pathways.

\section{Results}

Engraftment in Non-obese diabetic (NOD)/Severe combined immunodeficiency (SCID) mice

Engraftment occurred in 8 of 10 samples (80\%), producing disseminated human neoplastic lymphocytes in peripheral blood (PB), and bone marrow or infiltrated organs. The median mouse survival duration was 57.3 days (range, 40 to 60 ). In most cases, a gradual increase in circulating neoplastic cells was seen; in some cases, no neoplastic cells were detected in peripheral blood and evidence of engraftment was obtained at necropsy. Jurkat cell like neoplastic cells were found in $\mathrm{PB}$, bone marrow smear (Figure 1 top panel showed one representative bone marrow smear, the neoplastic cells in the T-ALL) and infiltrated in other organs including liver, spleen, lung, kidney and gastro intestine. These results were confirmed with hematoxylin and eosin (H\&E) staining of mouse liver (representative data shown in Figure 1 low panel).

\section{Notch1 and Foxp3 gene and protein expression were higher} in $T$-ALL mice than normal mice

We assessed Notch1 and Foxp3 expression in PB in TALL mice and the control by RT-PCR. Both Notch1 and Foxp3 were detected in T-ALL group, while in the control group, Notch1 was not detected and the expression of Foxp3 was significantly lower than T-ALL group $(P<0.05)$ (Figure 2). We next assessed Notch1 and Foxp3 protein expression in different organs. Both Notch1 and Foxp3 protein were detected in organs in normal mice and $\mathrm{T}$ ALL mice. Foxp3 protein was detected mostly around tumor tissues (Figure 3). Notch1 and Foxp3 protein expression in T-ALL mice were significantly higher than the control $(P<0.05)$.

\section{Jurkat cells express Notch1 and more Foxp3 than normal PBMCs}

We assessed the expression of Notch1 in Jurkat cells and PBMCs from healthy donors by RT-PCR and western-blot. Jurkat cells expressed Notch1. The expression of Notch1Cleaved protein was $48.03 \pm 1.57 \%$ by western-blot. We

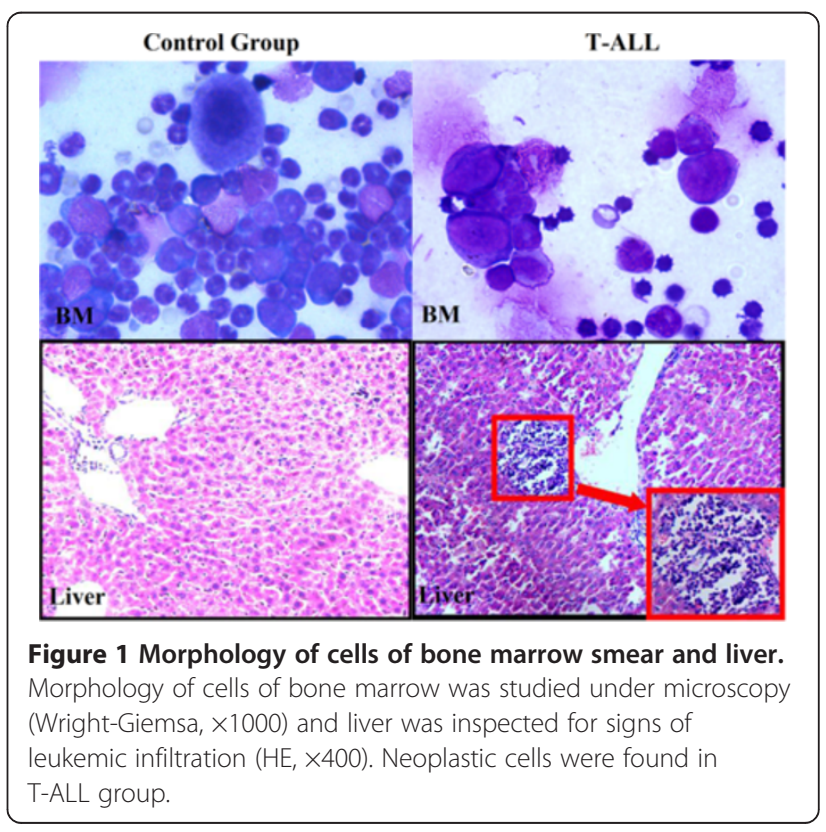




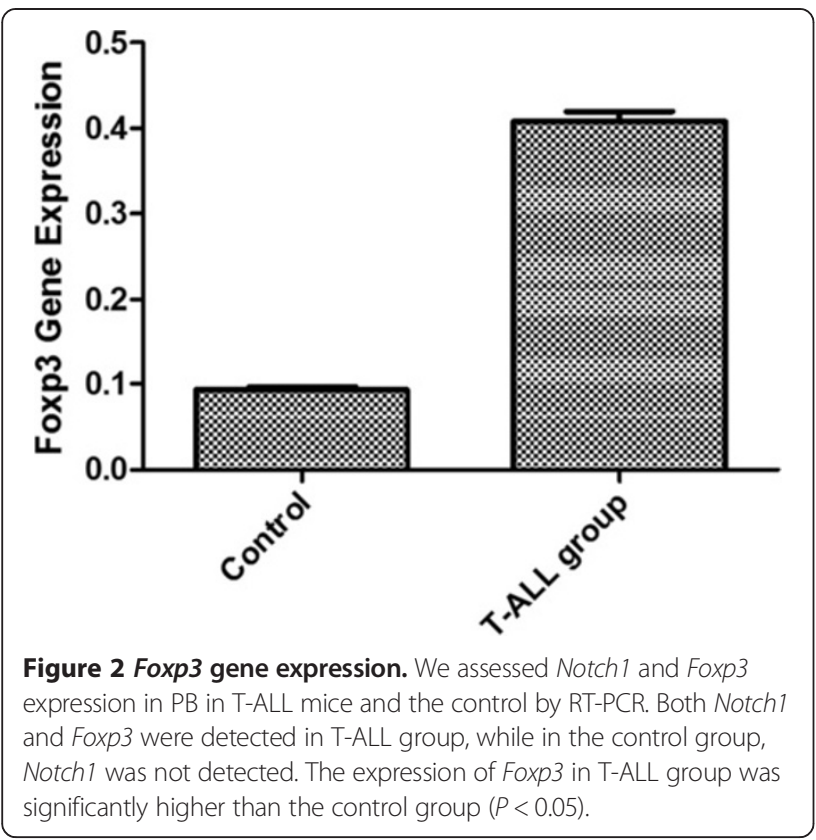

also assessed the expression of Foxp3 in Jurkat cells and PBMCs by Real-time PCR and flow cytometry. As shown in Figure 4, Foxp3-expressing jurkat cells were $88 \pm 2.5 \%$, which is much more than Foxp3-expressing PBMCs $(5 \pm 3.5 \%)(P<0.05)$.

\section{Blocking Notch1 signal by DAPT inhibits the proliferation of Jurkat cells}

To study the characteristics of Jurkat cells after DAPT treatment for 48 hours, cells were viewed under microscope. Jurkat cells without DAPT were usually round with clear areas of cytoplasm and nuclear and proliferated into cell clusters. However, Jurkat cells with DAPT were shown difficult to proliferate into cell clusters.

We next proved that DAPT could inhibit Jurkat cell proliferation by CCK8 method. Jurkat cells were treated with increasing concentrations of DAPT $(1,2.5,5,10$, $20 \mu \mathrm{M})$ for $4,8,12,24,48$ and 72 hours, respectively. After stimulated for 4,8 and 12 hours, Jurkat cells proliferated as those treated with DMSO alone. Jurkat cell proliferation was inhibited more and more remarkably as the concentration of DAPT increased after they were stimulated for 24 and 48 hours compared to DMSO control. However, after 72 hours stimulation, the proliferation of Jurkat cells was not inhibited by DAPT. These results indicated that DAPT could inhibit Jurkat cell proliferation only after 24 and 48 hours stimulation, especially the 48-hour time point and the inhibition was in a concentration-dependent manner with the greatest effect observed at a concentration of $20 \mu \mathrm{M}$, and the inhibition rate was as high as $33 \pm 2.3 \%$ (Figure 5 ).

To study the effect of DAPT on cell cycle, we further stimulated Jurkat cells with increasing concentrations of DAPT $(1,5,10,20 \mu \mathrm{M})$ for 48 hours. The results showed that the percentage of Jurkat cells in the subG0/G1 phase increased significantly while in S and G2/M phase decreased $(P<0.05)$. Increasing concentrations of DAPT induced G0/G1 phase cell cycle arrest in more Jurkat cells, indicative of apoptosis (Figure 6).

\section{Blocking Notch1 signal by DAPT induces Jurkat cells apoptosis}

To further document the effects of DAPT on apoptosis, analysis by annexin V/PI staining was performed after treatment with increasing concentrations of DAPT $(1,5$, $10,20 \mu \mathrm{M})$. The results showed an increase in apoptotic cells in Jurkat cells as the concentration of DAPT increased. The apoptosis rate with DAPT $(1,5,10,20 \mu \mathrm{M})$ was $21.7 \pm 2.77 \%, 22.7 \pm 2.71 \%, 37.3 \pm 4.9 \%$ and $33.7 \pm 4 \%$, respectively, compared with $0.84 \pm 0.38 \%$ for control (Figure 7) $(P<0.05)$.

\section{Notch1 and Hes-1 gene and protein expression is down regulated}

Jurkat cells were treated with increasing concentrations of DAPT $(1,5,10,20 \mu \mathrm{M})$ for 48 hours and RT-PCR was used to assess Notch1 gene expression. Notch1 was down regulated in Jurkat cells with DAPT treatment compared with cells with DMSO.

Hes 1 is one of the target genes of Notch1 signal. RealTime PCR was used to assess Hes-1 expression. Hes 1 was down regulated in Jurkat cells treated with $10 \mu \mathrm{M}$ DAPT for 24, 48 and 72 hours and gene expression was $53.59 \pm 12.7 \%, 28.95 \pm 4.2 \%$ and $27.35 \pm 1.4 \%$, respectively, compared to the control $(P<0.05)$. Hes 1 expression had a significant decrease after 48 hours treatment with DAPT (Figure 8A). At this time point, Hes1 expression was $90.12 \pm 1.4 \%, 57.3 \pm 2.2 \%, 42.1 \pm 3.3 \%$ and $41.8 \pm$ $6 \%$, respectively, in Jurkat cells with different concentrations of DAPT $(1,5,10,20 \mu \mathrm{M})$ compared to the control $(P<0.05)$. DAPT had the greatest effect on Hes 1 expression when its concentrations were $10 \mu \mathrm{M}$ (Figure 8B). We next sought to assess the Notch1-Cleaved and Hes-1 protein by western blot. At 48 hours treatment with $10 \mu \mathrm{M}$ DAPT, Notch1-Cleaved and Hes-1 protein expression was $72.5 \pm 3.8 \%$ and $32.1 \pm 2.9 \%(P<0.05)$, respectively, which was lower than the control group. Therefore, DAPT can inhibit Notch1-Cleaved and Hes-1 protein expression (Figure 8C).

\section{In vitro DAPT treatment block Foxp3 expression}

As reported by Ouyang, Notch 1 signaling can activate the Foxp3 promoter. We then assessed Foxp3 gene and protein expression after Notch1 inhibition. Foxp3 expression was $89 \pm 2.1 \%, 67.3 \pm 3 \%, 46.98 \pm 2.5 \%$ and $45 \pm 3.2 \%$ when DAPT was at 1, 5, 10 and $20 \mu \mathrm{M}$, respectively. Foxp3 expression was down regulated as the concentrations of 


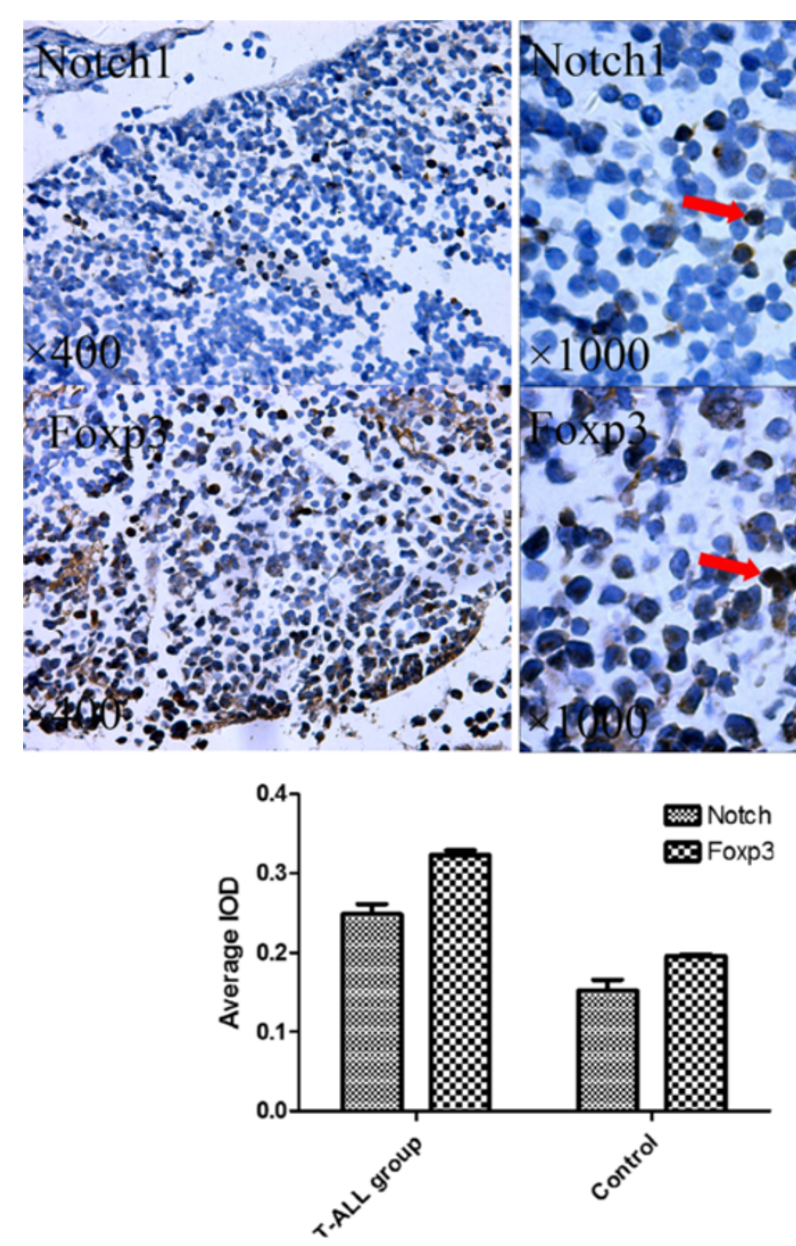

Figure 3 Notch1 and Foxp3 protein expression in tumor tissues. Tumor tissues in T-ALL mice were collected immunohistochemical assay. Samples were treated with rabbit polyclonal anti-Notch1 and anti-Foxp3. Both Notch1 and Foxp3 protein were detected in T-ALL mice and Foxp3 protein was detected mostly around tumor tissues. Image-pro plus was used to evaluate the expressions of Notch1 and Foxp3 through immunohistochemical staining. Protein expression was measured in IOD. Notch1 and Foxp3 protein expression in T-ALL mice were significantly higher than the control $(P<0.05)$.



Figure 4 Foxp3 expression in Jurkat cells and PBMCs. The expression of Foxp3 in Jurkat cells and PBMCs was analyzed by flow cytometry. Foxp3-expressing jurkat cells were much more than Foxp3-expressing PBMCs $(P<0.05)$. 


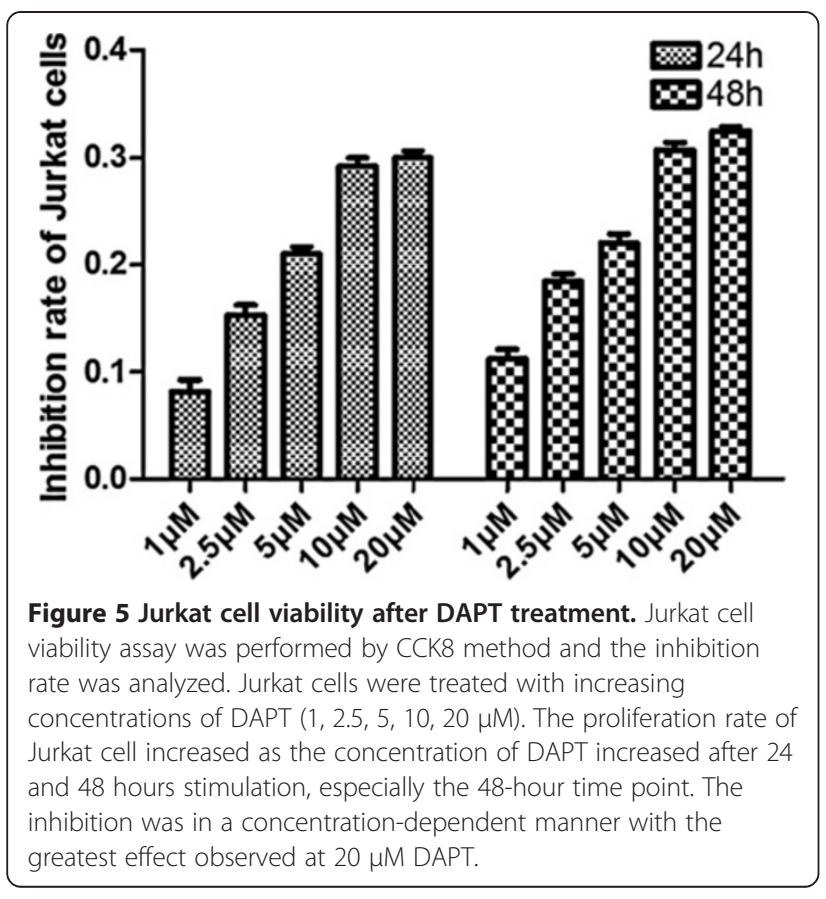

DAPT increased compared to the control $(P<0.05)$ (Figure 9A). Foxp3 expression was $90.5 \pm 6.7 \%, 46.98 \pm$ $2.5 \%$ and $112 \pm 14 \%(P<0.05)$ when Jurkat cells were treated with DPAT at $10 \mu \mathrm{M}$ for 24,48 and 72 hours, respectively. This showed that DAPT had the greatest effect on Foxp3 expression when Jurkat cells were treated with DAPT at $10 \mu \mathrm{M}$ for 48 hours. In contrast, after

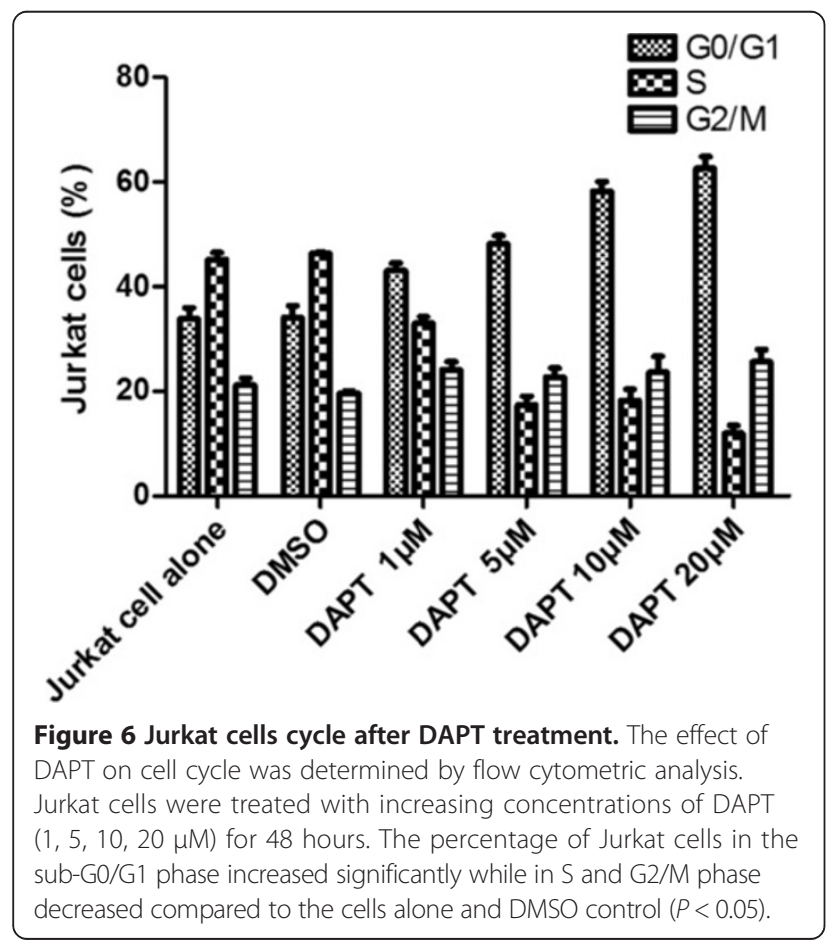

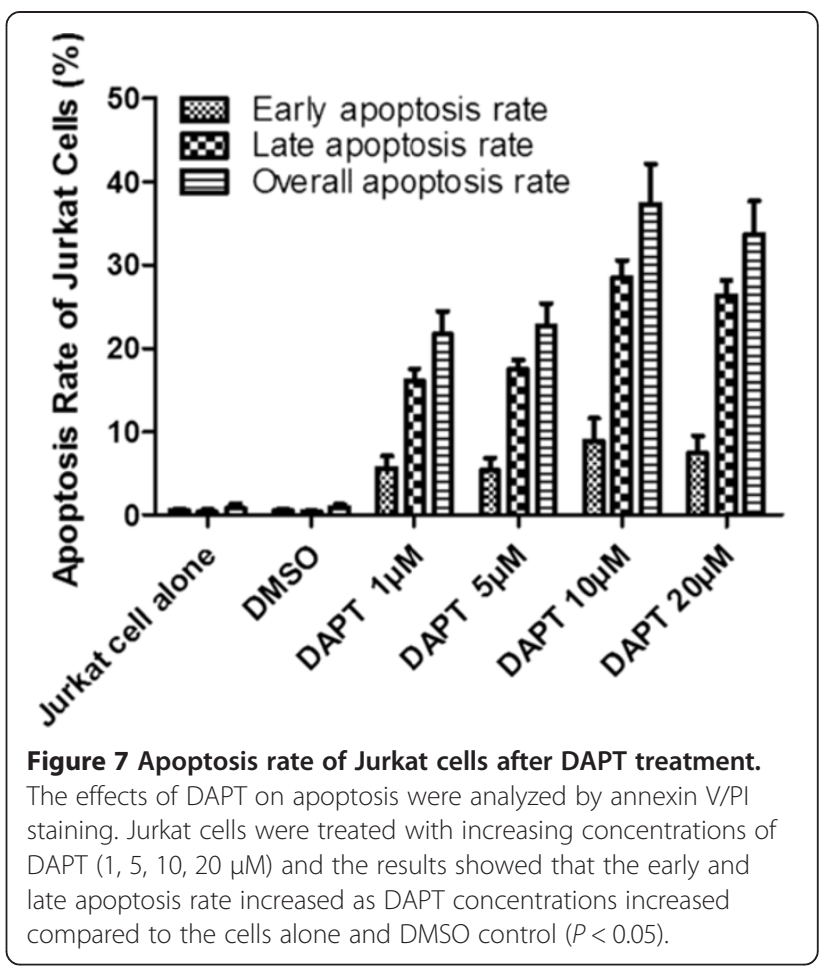

72 hours, Foxp3 expression was up regulated (Figure 9B). Flow cytometry was used to assess the Foxp3 protein expression and the result showed that DAPT could also inhibit Foxp3 protein expression. Foxp3 protein expression was $65.5 \pm 3.5 \%, 60.9 \pm 2.4 \%, 58.8 \pm 2.8 \%$ and $50.7 \pm$ $1.9 \%$ when Jurkat cells were treated with DAPT $(1,5$, $10,20 \mu \mathrm{M}$ ) for 48 hours. Similar to the gene expression, Foxp3 protein expression began to increase at 72 hours treatment with $10 \mu \mathrm{M}$ and $20 \mu \mathrm{M}$ DAPT. This inhibition effect was similar to what was observed in Jurkat cells, which began to proliferate after 72 hours treatment with DAPT.

\section{The expression of NF-KB, $p$-ERK $1 / 2$ and STAT1 are} deregulated in Jurkat cells after Notch1 signal inhibition $p$-ERK1/2, STAT1 and NF- $\kappa B$ are Notch1 target genes. To determine whether Notch1 inhibition was related to the expression of $\mathrm{p}$-ERK1/2, STAT1 and NF- $\mathrm{kB}$, we assessed the protein expression after Notch1 inhibition by DAPT. Similar to what was observed in Notch1, Hes-1 and Foxp3 expression, p-ERK1/2, STAT1 and NF-kB protein expression was down regulated when Jurkat cells were treated with $10 \mu \mathrm{M}$ DAPT for 48 hours. p-ERK1/2, STAT1 and NF- $\mathrm{kB}$ protein expression was $50.1 \pm 2.9 \%, 68.8 \pm 3.8 \%$ and $48.7 \pm 1.4 \%$, respectively $(P<0.05)$ (Figure 10$)$.

\section{Discussion}

Foxp ${ }^{+}$Tregs play an important role in regulating the immune system by suppressing self-reactive $\mathrm{T}$ cells that have escaped negative selection in the thymus as well as 

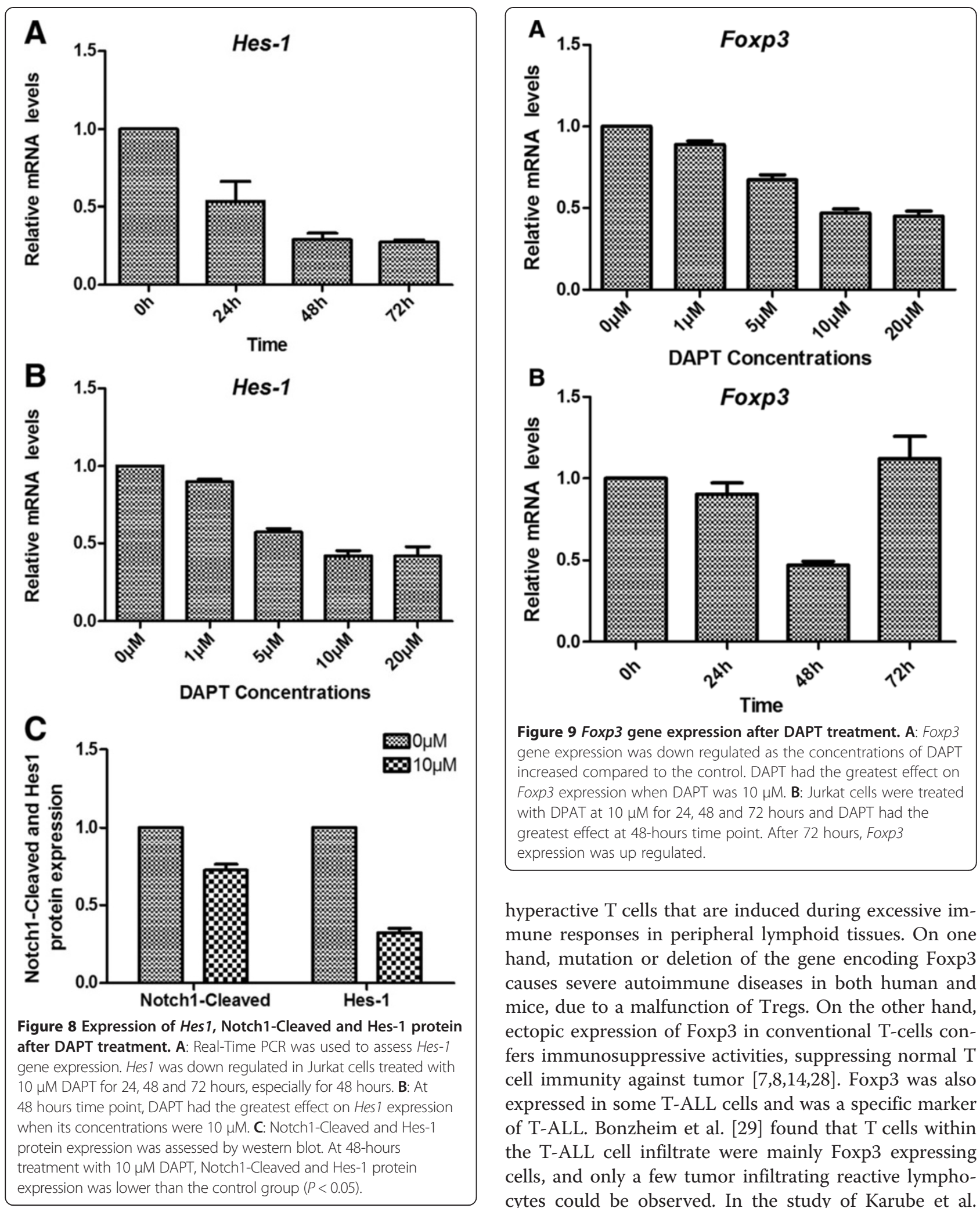

hyperactive $\mathrm{T}$ cells that are induced during excessive immune responses in peripheral lymphoid tissues. On one hand, mutation or deletion of the gene encoding Foxp3 causes severe autoimmune diseases in both human and mice, due to a malfunction of Tregs. On the other hand, ectopic expression of Foxp3 in conventional T-cells confers immunosuppressive activities, suppressing normal $\mathrm{T}$ cell immunity against tumor $[7,8,14,28]$. Foxp3 was also expressed in some T-ALL cells and was a specific marker of T-ALL. Bonzheim et al. [29] found that T cells within the T-ALL cell infiltrate were mainly Foxp3 expressing cells, and only a few tumor infiltrating reactive lymphocytes could be observed. In the study of Karube et al. [7], Foxp3 expression was confirmed in T-ALL and Foxp3 ${ }^{+}$ T-ALL cells might suppress tumor immunity and promote tumor growth. Roncador et al. [30] also reported that Foxp $^{+}$T-ALL showed a more aggressive clinical course 


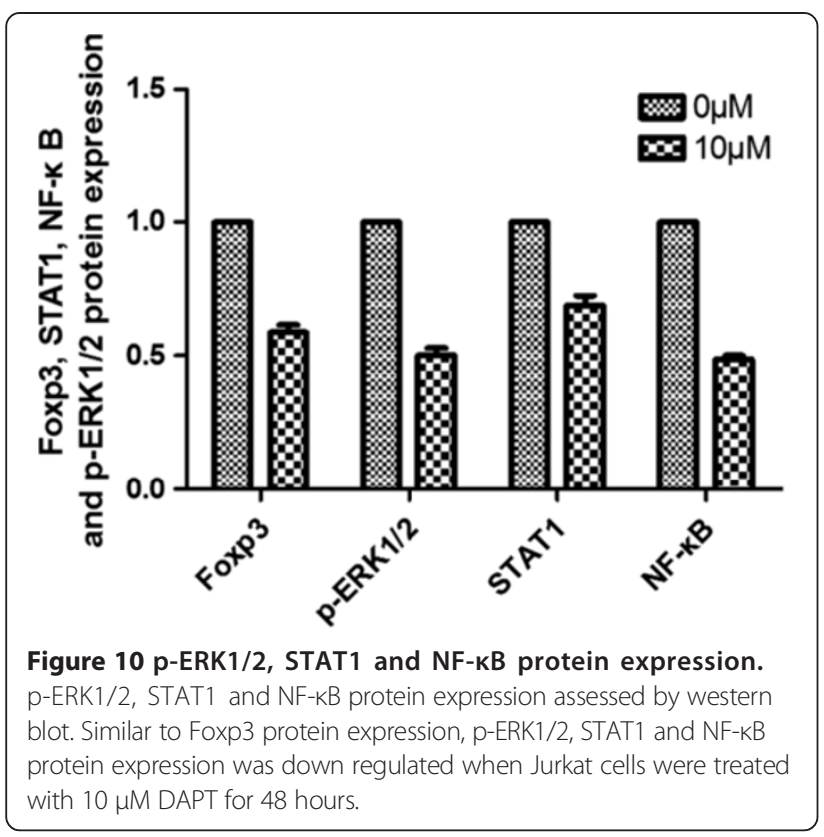

than Foxp3- T-ALL. In our study, we established T-ALL murine model with SCID mice and found that Foxp3 expression increased in T-ALL mice compared to normal mice. We then detected Foxp3 expression in both human $\mathrm{T}$ cell leukemia cell line and PBMCs from healthy donors. We found that Foxp3 expression was higher in Jurkat cells than in PBMCs. The results from in vivo and in vitro indicated that Foxp3 expression was associated with T-ALL, which was compatible with what was found in Karube's study.

Recently, deregulation of Notch signaling has been linked to the development of T-ALL. The recent identification of activating mutations in Notch1 in the majority of T-ALL has brought major interest towards targeting the Notch signaling pathway in this disease $[3,12,15,16,22,31,32]$. The fundamental importance of Notch1 mutations in T-ALL is highlighted by the potential role of Notch1 as a molecular therapeutic target for the treatment of this disease. Pharmacologic inhibition effectively abrogates oncogenic Notch1 signaling in T-ALL cells. GSIs induced rapid clearance of intracellular activated Notch1 protein and transcriptional downregulation of Notch1 target genes [1,3,4,11]. In our study, the biological characteristics of Jurkat cells as well as Notch1 target gene expression were studied after pharmacologic inhibition of Notch signaling using GSI. DAPT inhibited the proliferation of Jurkat cells. As DAPT concentrations increased, the viability of Jurkat cells decreased. DAPT induced G0/G1 phase cell cycle arrest in Jurkat cells, which resulted in apoptosis. We further detected Notch1 and Hes-1 gene and protein expression after DAPT treatment. Notch1 and Hes-1 were down regulated and Notch1-Cleaved and Hes-1 protein expression significantly decreased compared to the control group. These suggested that DAPT could inhibit Notch1 signaling by down regulating Notch1 target genes and induce Jurkat cell apoptosis.

Except for the aberrant Notch mutation that induces T-ALL, immunosuppression in T-ALL has also been the subject of many discussions. Karube et al. [7] indicated that T-ALL cells might function as Treg-like cells and induce the immunosuppressive state especially in Foxp $3^{+}$ cases. However, the mechanisms leading to immune tolerance by Foxp $3^{+}$Tregs in T-ALL remain largely unknown. Recently, Notch and its ligands have been implicated in the regulation and differentiation of various $\mathrm{CD} 4^{+} \mathrm{T}$-helper cells $[2,3,9-11,33]$. Is Notch1 also involved in regulating Foxp3? Samon et al. [34] provided evidences that Foxp3 was a downstream target of Notch signaling. Pharmacologic inhibition of Notch signaling using GSIs blocked the up-regulation of Foxp3 target genes and induces Foxp3 expression [34]. GSIs also inhibited the binding of Notch1, CSL, and Smad to conserved binding sites in the Foxp3 promoter. Moreover, in vivo GSIs treatment down-regulated Foxp3 expression and resulted in a spontaneous lymphocyte infiltration of the liver in mice. Ou-Yang et al. [8] showed that Notch signaling could modulate the Foxp3 promoter through RBP-J- and Hes1dependent mechanisms and Notch signaling might be involved in the development and function of Tregs through regulating Foxp3 expression. In order to study the association between Notch1 and Foxp3, we detected Foxp3 gene and protein expression in Jurkat cells treated with DAPT. Notch1 and Hes-1 had a significant drop and Foxp3 was down regulated at the same time point. This suggested that Notch1 signaling was involved in regulating Foxp3 expression in Jurkat cell.

These previous findings led us to explore the crosstalk between Notch1 and Foxp3 in Jurkat cells. We hypothesized that activated Notch1 might increase Foxp3 expression by up regulating some target genes. Previous reports [25-27,35,36] have suggested that Notch can display both stimulatory and inhibitory control of NF- $\mathrm{kB}$ activity. It has been hypothesized that activated Notch in $\mathrm{T}$ cells may result in constitutive NF- $\mathrm{kB}$ activation, leading to T-cell leukemia/lymphoma. $N F-\kappa B$ as well as $p$-ERK1/2 and STAT1 are Notch 1 target genes. We assessed the protein expression of NF-kB, p-ERK1/2 and STAT1. The result showed that the protein expression was down regulated after Notch1 was inhibited by DAPT. These suggested that inhibition of Foxp3 expression involved Notch signaling, and it may be mediated by regulation of NF-kB, p-ERK1/2 and STAT1 pathways.

\section{Conclusions}

In summary, this study systematically showed Notch 1 and Foxp3 expression as well as its impact on T-ALL cell proliferation and development. By studying the biological 
change of Jurkat cells after Notch1 inhibition, we showed that down regulation of Notch1 and Foxp3 could induce Jurkat cell apoptosis. The association between Notch1 and Foxp3 was another important subject of this study. Notch signaling is involved in regulating Foxp3 expression in Jurkat cells and it could be mediated by regulation of NF- $\mathrm{B}$, p-ERK1/2 and STAT1 pathways. These results together indicated that Notch1 signaling that induces Foxp3 expression might be associated with immunosuppression state in T-ALL.

\section{Materials and methods}

\section{Ethics statement}

Peripheral blood samples in this study were collected from healthy donors in hospital. All participants are residence in our country. Samples were collected for diagnostic purposes. After the original purpose has been achieved, the residual samples were used for research only without additional charges. All participants were informed of full information about the purposes of the sampling, and/or the plan of the research proposal. All participants have signed the informed consent before enrolling in this study. The informed consent is not only for this study, but also for other studies in which human blood samples are needed. All signed consent is in Chinese and documented. This ethics approval was obtained from Committee on the Ethics of the First Affiliated Hospital of Guangzhou Medical College (Permit number: 2012-41).

This study (Establishment of T-cell acute lymphoblastic leukemia murine model using NOD/SCID mice and the assessment of Notch1 and Foxp3 expression in this model) was carried out in strict accordance with the recommendations in the Guide for the Care and Use of Laboratory Animals of Guangzhou Medical College. Animals were purchased from Animal experimental center, Guangdong, China. The protocol was approved by the Committee on the Ethics of the First Affiliated Hospital of Guangzhou Medical College (Permit number: 2012-41). Mice that developed T-ALL may have experienced discomfort. Signs included increased abdominal girth from tumor infiltration, dehydration, decreased activity and cachexia. Mice with T-ALL were susceptible to infection. Mice were observed daily by laboratory staff and animal technicians and weighed once a week to detect weight loss. If the mice decompensated, they were immediately euthanized by $\mathrm{CO}_{2}$ to minimize suffering.

\section{Cell line and samples}

Jurkat cells are a human $\mathrm{T}$ cell leukemia cell line that constitutively expresses IC [37] and, therefore, were used in this study. Jurkat cells were purchased from American Type Cell Culture (ATCC) and maintained according to the ATCC protocol. As described elsewhere [38], peripheral blood mononuclear cells (PBMCs) were separated from fresh blood samples by density gradient centrifugation. Red blood cells were removed from splenocytes using ammonium chloride lysis buffer.

\section{Experimental animal and procedures}

NOD/ SCID mice (Animal experimental center, Guangdong, China) were used. Twenty female mice aged 5 weeks were maintained in a specific pathogen-free environment. Twenty mice weight $10.45 \mathrm{~g}$ to $11.62 \mathrm{~g}$ (median weight $11.12 \mathrm{~g}$ ) were divided into T-ALL group and the control group randomly with 10 mice in each group. Physical randomisation procedure using random number tables was performed to assign mice to each group. Mice were injected intraperitoneally with cyclophosphamide $(100 \mathrm{mg} / \mathrm{kg} / \mathrm{d})$ [39] for 2 days. In T-ALL group, Jurkat cells in the logarithmic phase of growth were then collected and transferred intravenously $\left(5 \times 10^{6} /\right.$ mouse/day) through tail vein for 2 days. Mice in the control group were injected with physiological saline. Engraftment of Jurkat cells in mice was monitored by serial tail vein sampling every 7 days. This was done without anesthesia. To warm the tail with the aid of a heat lamp to increase obtainable blood volume before tail nicking. Decompensated mice were euthanized by $\mathrm{CO}_{2}$, when $\mathrm{PB}$ infiltration or clinical status like suggested engraftment. Mice were exposed to a $\mathrm{CO}_{2}$ concentration of $70 \%$ and maintained for 2 minutes after apparent clinical death. Other mice were evaluated for 60 days before sacrifice and necropsy. PB was collected for Notch1 and Foxp3 gene expression. Internal organs were inspected for signs of leukemic infiltration. Tissues from infiltrated organs were collected for Notch1 and Foxp3 protein expression. Single-cell suspensions from bone marrow were also prepared for flow cytometric analysis.

\section{Histopathology and immunochemistry}

Samples of tissues were immersed in 10\% neutral formalin. Formalin-preserved specimens were then embedded in paraffin, cut into $5 \mu \mathrm{m}$ sections, and stained with H\&E for histopathology examination. For immunohistochemical assay, paraffin-embedded sections were dewaxed, rehydrated and incubated with $0.5 \%$ hydrogen peroxide in methanol to quench endogenous tissue peroxidase. Sections were incubated with pepsin for 45 min for antigen retrieval. After blocking nonspecific sites with 1\% BSA in PBS, sections were treated with rabbit polyclonal antiNotch1 and anti-Foxp3 (Abcam) overnight and then with appropriate biotin-conjugated secondary antibodies for $20 \mathrm{~min}$. Image-pro plus was used to evaluate the expressions of Notch1 and Foxp3 using immunohistochemical staining. Protein expression was measured in integrated optical density (IOD). 


\section{Reverse-transcription PCR (RT-PCR) and real-time PCR}

Total RNA was isolated from 1-5 $\times 10^{6}$ Jurkat cells using the RNeasy kit (Qiagen) and was resuspended in $40 \mu \mathrm{l}$ RNase free $\mathrm{H}_{2} \mathrm{O}$. First-strand cDNA synthesis was performed with oligo (dT) as primer. Notch1-IC primers were $5^{\prime}$-TTCCCTGAGGGCTTCAAAGT-3' (forward) and 5'-CCCGCTACTCACGCTCTG-3' (reverse). The primers of extracellular domain of Notch1 were 5 '-CCGGTGAGA CCTGCCTGAAT-3' (forward) and 5'-GCACTTGTACTC CGTCAGCG-3' (reverse). RT-PCR for Notch1 was performed in duplicate $\left(30\right.$ cycles of $98^{\circ} \mathrm{C}$ for $10 \mathrm{~s}, 55^{\circ} \mathrm{C}$ for $30 \mathrm{~s}$, and $72^{\circ} \mathrm{C}$ for $45 \mathrm{~s}$ ). PCR products were subjected to $2 \%$ agarose gel electrophoresis and relative gene expression was measured in grey value. Foxp3 primers were $5^{\prime}$-ACTGACCAAGGCTTCATCTGTG-3' (forward) and 5'-GGAACTCTGGGAATGTGCTGT-3' (reverse). RTPCR for Foxp3 mRNA expression (in vivo experiment) was performed as before. Real-time PCR for Foxp3 mRNA quantification was performed in duplicate with the Sofast EvaGreen Supermix (Bio-Rad) $\left(40\right.$ cycles of $95^{\circ} \mathrm{C}$ for $30 \mathrm{~s}$, $95^{\circ} \mathrm{C}$ for $5 \mathrm{~s}$, and $56^{\circ} \mathrm{C}$ for $10 \mathrm{~s}, 65-95^{\circ} \mathrm{C}$ for $10 \mathrm{~s}$ ). Hes- 1 primers were $5^{\prime}$-GGCTAAGGTGTTTGGAGGCT-3' (forward) and $5^{\prime}$-GCTGTTGCTGGTGTAGACGG-3' (reverse). Real-time PCR was performed as before.

\section{Western blotting}

Cells were lysed in RIPA buffer with a protease inhibitor mixture and a phosphatase inhibitor mixture (Shanghai Biocolors); and lysates were run on 10\% SDS-polyacrylamide gels. After transfer, the polyvinyl difluoride membranes (Millipore) were blocked for $1 \mathrm{~h}$ with TBS/Tween 20 containing $5 \%$ powder skim milk and then probed overnight at $4^{\circ} \mathrm{C}$ with primary $\mathrm{Ab}$ specific for cleaved Notch 1 (rabbit anti-human IgG, ABCAM). Blots were then washed five times and probed for $1 \mathrm{~h}$ with secondary $\mathrm{Ab}$ (goat anti-rabbit IgG, ABCAM). Membranes were developed with Immobilon Western Chemiluminescent HRP substrate (Millipore).

\section{Flow cytometry}

Jurkat cells were co-cultured with DAPT for 48 hours and stained with fluorochrome-labeled mAbs against Foxp3 (eBioscience). Intracellular Foxp3 staining was performed using the Cytofix/Cytoperm intracellular staining kit, according to the manufacturer's instructions. Flow cytometry was performed with Epics XL system (Beckman Courter) and analyzed using Expo 32 software.

\section{Cell viability assay}

The number of viable cells was determined using a Cell Counting Kit- 8 assay according to the manufacturer's instructions (Dojindo, Japan). Cells were plated at a density of $3 \times 10^{4}$ cells per well in a 96-well plate. After incubation for 6 hours, DAPT was added to each well at
$1,2.5,5,10$ and $20 \mu \mathrm{M}$. Cells treated with $0.1 \%$ DMSO as control. After incubated for 4, 8, 12, 24, 48 and 72 hours, cells were incubated with kit reagent WST-8 for a further $2 \mathrm{~h}$. The absorbance of samples $(450 \mathrm{~nm})$ was determined using a scanning multiwell spectrophotometer that serves as an ELISA reader.

\section{Cell cycle analysis}

The cell cycle distribution was determined by flow cytometric analysis. Cells were re-suspended into $5 \times$ $10^{5}$ cells $/ \mathrm{ml}$ and incubated with DAPT $(1,5,10$ and $20 \mu \mathrm{M})$ for 48 hours. Then cells were collected and nuclear staining was performed according to the manufacturer's instructions using Flow Cytometry Analysis of Cell Cycle Kit (GENMED, Shanghai). Following staining, cells were immediately analyzed by flow cytometry.

\section{Apoptosis analysis}

Jurkat cells were stained with Wright-Giemsa and morphology was studied under microscopy. Apoptosis induction was confirmed using the Annexin V/PI Apoptosis Detection Kit (Jingmei Biotech, Shanghai, China). After co-cultured with DAPI, Jurkat cells were collected and washed twice with cold PBS. Cells were labeled with $5 \mu \mathrm{l}$ Annexin VFITC followed by10 $\mu$ l PI. Annexin V-PI were measured by FACS Calibur and analyzed with the Modfit Software.

\section{Statistical analysis}

Data are expressed as mean \pm SD. Statistical significance was valued by one-way ANOVA. Equal variances assumed were LSD. A $P$ value less than .05 was considered statistically significant (SPSS 13.0 for windows, SPSS Inc, Chicago, IL).

\section{Competing interests}

The authors declare that they have no competing interests.

\section{Authors' contributions}

XL has made substantial contributions to conception and design, and has been involved in drafting and revising the manuscript. HT have given final approval of the version to be published. $Y Z$ has participated in in vitro study and the analysis of data. TX has participated in in vivo study and the analysis of data. CW has been involved in data analysis. YL has been involved in

revising the manuscript. All authors read and approved the final manuscript.

\section{Acknowledgement}

We thank Baodan Yu and Lixia Zheng for valuable technical assistance. The study was supported by grants from the National Natural Science

Foundation of China (No. 81200399/H0813)

\section{Author details}

'Department of Oncology \& Hematology, the First Affiliated Hospital of Guangzhou Medical College, Guangzhou 510230, China. ${ }^{2}$ Institute of Hematology, Medical College, Jinan University, Guangzhou, China.

Received: 26 January 2013 Accepted: 2 April 2013

Published: 11 April 2013

\section{References}

1. Ferrando A: The role of NOTCH1 signaling in T-ALL. Hematology Am SoC Hematol Educ Program 2009, 2009:353-361. 
2. Palomero T, Ferrando A: Therapeutic targeting of NOTCH1 signaling in T-ALL. Clin Lymphoma Myeloma 2009, 9(Suppl 3):S205-S210.

3. Aster JC, Blacklow SC, Pear WS: Notch signalling in T-cell lymphoblastic leukaemia/lymphoma and other haematological malignancies. J Pathol 2011, 223:262-273.

4. Real PJ, Ferrando AA: NOTCH inhibition and glucocorticoid therapy in T-cell acute lymphoblastic leukemia. Leukemia 2009, 23:1374-1377.

5. Trioche P, Nelken B, Michel G, Pellier I, Petit A, Bertrand Y, Rohrlich P, Schmitt C, Sirvent N, Boutard P, Margueritte G, Pautard B, Ducassou S, Plantaz D, Robert A, Thomas C, Desseaux K, Chevret S, Baruchel A: French "real life" experience of clofarabine in children with refractory or relapsed acute lymphoblastic leukaemia. Exp Hematol Oncol 2012, 1:39.

6. Zou L, Zhang H, Du C, Liu X, Zhu S, Zhang W, Li Z, Gao C, Zhao X, Mei M, Bao S, Zheng H: Correlation of SRSF1 and PRMT1 expression with clinical status of pediatric acute lymphoblastic leukemia. J Hematol Oncol 2012, 5:42.

7. Karube K, Aoki R, Sugita Y, Yoshida S, Nomura Y, Shimizu K, Kimura Y, Hashikawa K, Takeshita M, Suzumiya J, Utsunomiya A, Kikuchi M, Ohshima K: The relationship of FOXP3 expression and clinicopathological characteristics in adult T-cell leukemia/lymphoma. Mod Pathol 2008 21(5):617-625

8. Ou-Yang HF, Zhang HW, Wu CG, Zhang P, Zhang J, Li JC, Hou LH, He F, Ti XY, Song LQ, Zhang SZ, Feng L, Qi HW, Han H: Notch signaling regulates the FOXP3 promoter through RBP-J- and Hes1-dependent mechanisms. Mol Cell Biochem 2009, 320(1-2):109-114.

9. Jundt F, Schwarzer R, Dorken B: Notch signaling in leukemias and lymphomas. Curr Mol Med 2008, 8(1):51-59.

10. Demarest RM, Ratti F, Capobianco AJ: It's T-ALL about notch. Oncogene 2008, 27:5082-5091.

11. Sandy AS, Maillard I: Notch signaling in the hematopoietic system. Expert Opin Biol Ther 2008, 9(11):1383-1398.

12. Lin C, Zheng H, Wang C, Yang L, Chen S, Li B, Zhou Y, Tan H, Li Y Mutations increased overexpression of Notch1 in T-cell acute lymphoblastic leukemia. Cancer Cell Int 2012, 12:13.

13. Dudley DD, Wang HC, Sun XH: Hes 1 potentiates $T$ cell lymphom agenesis by up regulating a subset of notch target genes. PLoS One 2009, 4(8):e6678.

14. Asano N, Watanabe T, Kitani A, Fuss IJ, Strober W: Notch1 signaling and regulatory T cell function. J Immunol 2008, 180:2796-2804.

15. Zou J, Li P, Lu F, Liu N, Dai JJ, Ye JJ, Qu X, Sun XL, Ma DX, Park J, Ji CY Notch1 is required for hypoxia-induced proliferation, invasion and chemoresistance of T-cell acute lymphoblastic leukemia cells. J Hematol Oncol 2013, 6:3.

16. Guo DF, Teng QL, Ji CY: NOTCH and phosphatidylinositide 3-kinase/ phosphatase and tensin homolog deleted on chromosome ten/AKT/ mammalian target of rapamycin (mTOR) signaling in T-cell development and T-cell acute lymphoblastic leukemia. Leuk Lymphome 2011, 52(7):1200-1210

17. Jarriault S, Brou C, Logeat F, Schroeter EH, Kopan R, Israel A: Signalling downstream of activated mammalian Notch. Nature 1995, 377:355-358.

18. Weng AP, Millholland JM, Yashiro-Ohtani Y, Arcangeli ML, Lau A, Wai C, Wai C, Del Bianco C, Rodriguez CG, Sai H, Tobias J, Li YM, Wolfe MS, Shachaf C, Felsher D, Blacklow SC, Pear WS, Aster JC: c-Myc is an important direct target of Notch1 in T-cell acute lymphoblastic leukemia/lymphoma. Genes Dev 2006, 20:2096-2109.

19. Palomero T, Lim WK, Odom DT, Sulis ML, Real PJ, Margolin A, Barnes KC, O'Neil J, Neuberg D, Weng AP, Aster JC, Sigaux F, Soulier J, Look AT, Young RA, Califano A, Ferrando AA: NOTCH1 directly regulates c-MYC and activates a feed-forward-loop transcriptional network promoting leukemic cell growth. Proc Natl Acad Sci 2006, 103:18261-18266.

20. Thompson BJ, Buonamici S, Sulis ML, Palomero T, Vilimas T, Basso G, Ferrando A, Aifantis I: The SCFFBW7 ubiquitin ligase complex as a tumor suppressor in T cell leukemia. J Exp Med 2007, 204:1825-1835.

21. Palomero T, Sulis ML, Cortina M, Real PJ, Barnes K, Ciofani M, Caparros E, Buteau J, Brown K, Perkins SL, Bhagat G, Agarwal AM, Basso G, Castillo M, Nagase S, Cordon-Cardo C, Parsons R, Zuniga-Pflucker JC, Dominguez M, Ferrando AA: Mutational loss of PTEN induces resistance to NOTCH1 inhibition in T-cell leukemia. Nat Med 2007, 13(10):1203-1210.

22. Jin BF, Shen HX, Lin SB, Li JL, Chen ZR, Griffin JD, Wu LZ: The mastermindlike 1 (MAML1) Co-activator regulates constitutive NF-kappaB signaling and cell survival. J Biol Chem 2010, 285(19):14356-14365.

23. Watanabe R, Wei L, Huang J: mTOR signaling, function, novel inhibitors, and therapeutic targets. J Nucl Med 2011, 52:497-500.
24. Paczesny S, Hanauer D, Sun Y, Reddy P: New perspectives on the biology of acute GVHD. Bone Marrow Transplant 2010, 45(1):1-11.

25. Yvon ES, Vigouroux S, Rousseau RF, E. Biagi E, Amrolia P, Dotti G, Wagner $\mathrm{HJ}$, Brenner MK: Overexpression of the Notch ligand, Jagged-1, induces alloantigen-specific human regulatory T cells. Blood 2003, 102:3815-3821.

26. Vigouroux S, Yvon ES, Wagner HJ, Biagi E, Dotti G, Sili U, Lira C, Rooney CM, Brenner MK: Induction of antigen-specific regulatory $T$ cells following overexpression of a Notch ligand by human B lymphocytes. J Virol 2003, 77:10872-10880

27. Kared H, Adle-Biassette H, Fois E, Masson A, Bach JF, Chatenoud L, Schneider E, Zavala F: Jagged2-expressing hematopoietic progenitors promote regulatory $\mathrm{T}$ cell expansion in the periphery through notch signaling. Immunity 2006, 25:823-834.

28. Fontenot JD, Gavin MA, Rudensky AY: Foxp3 programs the development and function of CD4 + CD25+ regulatory T cells. Nat Immunol 2003, 4:330-336.

29. Bonzheim I, Geissinger E, Tinguely M, Roth S, Grieb T, Reimer P, Wilhelm M Rosenwald A, Müller-Hermelink HK, Rüdiger T: Evaluation of FoxP3 expression in peripheral T-cell lymphoma. Am J Clin Pathol 2008, 130:613-619.

30. Roncador G, Garcia JF, Maestre L, Lucas E, Menarguez J, Ohshima K, Nakamura S, Banham AH, Piris MA: FOXP3, a selective marker for a subset of adult T-cell leukaemia/ lymphoma. Leukemia 2005, 19:2247-2253.

31. Cullion K, Draheim KM, Hermance N, Tammam J, Sharma VM, Ware C, Nikov G, Krishnamoorthy V, Majumder PK, Kelliher MA: Targeting the Notch1 and mTOR pathways in a mouse T-ALL model. Blood 2009, 113:6172-6181.

32. Kojima K, Shimanuki M, Shikami M, Kojima K, Shimanuki M, Shikami M, Samudio IJ, Ruvolo V, Corn P, Hanaoka N, Konopleva M, Andreeff M, Nakakuma H: The dual PI3 kinase/mTOR inhibitor PI-103 prevents p53 induction by $\mathrm{Mdm} 2$ inhibition but enhances $\mathrm{p} 53$-mediated mitochondrial apoptosis in p53 wild-type AML. Leukemia 2008, 22:1728-1736.

33. Kamstrup MR, Rahbek Gjerdrum LM, Biskup E, Lauenborg BT, Ralfkiaer E, Woetmann A, Ødum N, Gniadecki R: Notch1 as a potential therapeutic target in cutaneous T-cell lymphoma. Blood 2010, 116:2504-2512.

34. Samon JB, Champhekar A, Minter LM, Telfer JC, Miele L, Fauq A, Das P, Golde TE, Osborne BA: Notch1 and TGF $\beta 1$ cooperatively regulate Foxp3 expression and the maintenance of peripheral regulatory T cells. Blood 2008, 112:1813-1821.

35. Vilimas T, Mascarenhas J, Palomero T, Mandal M, Buonamici S, Meng F, Thompson B, Spaulding C, Macaroun S, Alegre ML, Kee BL, Ferrando A, Miele L, Aifantis I: Targeting the NF-kappaB signaling pathway in Notch1induced T-cell leukemia. Nat Med 2007, 13(1):70-77.

36. Shin HM, Minter LM, Cho OH, Gottipati S, Faug AH, Golde TE, Sonenshein GE, Osborne BA: Notch1 augments NF-kappaB activity by facilitating its nuclear retention. EMBO J 2006, 25(1):129-138.

37. O'Neil J, Grim J, Strack P, Rao S, Tibbitts D, Winter C, Hardwick J, Welcker M, Meijerink JP, Pieters R, Draetta G, Sears R, Clurman BE, Look AT: FBW7 mutations in leukemic cells mediate NOTCH pathway activation and resistance to $\gamma$-secretase inhibitors. J Exp Med 2007, 204:1813-1824.

38. Devereux G, Hall AM, Barker RN: Measurement of T-helper cytokines secreted by cord blood mononuclear cells in response to allergens. J Immunol Methods 2000, 234:13-22.

39. Lin YP, Yang YP, Huang WM, Chen YH, Li SF, Fan YM: Experimental pathogenicity of a clinical isolate of Trichosporon dermatis in a murine model. Mycopathologia 2011, 172(5):381-387.

doi:10.1186/1475-2867-13-34

Cite this article as: Luo et al: Notch1 signaling is involved in regulating Foxp3 expression in T-ALL. Cancer Cell International 2013 13:34. 